\title{
Localized-Magnon Chains and Interchain Interactions
}

\author{
O. Krupnitska ${ }^{a}$, J. Richter ${ }^{b}$ And O. DeRzhKO ${ }^{a, c, b, d, *}$ \\ ${ }^{a}$ Institute for Condensed Matter Physics, National Academy of Sciences of Ukraine, \\ Svientsitskii Str. 1, 79011 L'viv, Ukraine \\ ${ }^{b}$ Institut für Theoretische Physik, Otto-von-Guericke-Universität Magdeburg, \\ P.O. Box 4120, 39016 Magdeburg, Germany \\ ${ }^{c}$ Department for Theoretical Physics, Ivan Franko National University of L'viv, \\ Drahomanov Str. 12, 79005 L'viv, Ukraine \\ ${ }^{d}$ Abdus Salam International Centre for Theoretical Physics, Strada Costiera 11, 34151 Trieste, Italy
}

(Received May 3, 2016)

We consider the spin-1/2 antiferromagnetic $X X Z$ Heisenberg model on a two-dimensional network of weakly coupled frustrated diamond chains, i.e., a spin model related to the celebrated azurite compound. Using the strongcoupling approach we construct an effective Hamiltonian which yields the low-temperature high-field properties of the spin system. We examine effects of interchain interactions on localized-magnon physics.

DOI: 10.12693/APhysPolA.132.1234

PACS/topics: localized magnons, interchain interaction, azurite

\section{Introduction}

There is a wide class of frustrated quantum Heisenberg antiferromagnets which exhibit the so-called localizedmagnon physics, i.e., they have spin excitations which are located within a small part of the lattice (trap) [1]. The existence of localized states is related to a completely dispersionless (flat) lowest-energy onemagnon band. Localized-magnon states dominate lowtemperature thermodynamics at high magnetic fields. By mapping onto a classical hard-core lattice gas, corresponding thermodynamic quantities can be calculated by classical statistical mechanics [2]. For a recent review on localized magnons see Ref. [3].

Among real-life compounds, there are several candidates which are expected to show localized-magnon physics. A natural mineral azurite, $\mathrm{Cu}_{3}\left(\mathrm{CO}_{3}\right)_{2}(\mathrm{OH})_{2}$, is the most prominent one [4]. A basic model for this compound is a frustrated spin- $1 / 2$ antiferromagnetic Heisenberg chain of "distorted diamond" geometry [4-6]. Furthermore, a small anisotropy of the exchange Heisenberg interaction may be expected and a full three-dimensional model must take into account (weak) interchain interactions. Thus, to provide theoretical predictions for, say, low-temperature high-field magnetization curve one has to extend the localized-magnon description taking into consideration deviations from the ideal flat-band geometry, exchange interaction anisotropy, and interchain interactions. In our previous studies we examined effects of nonideal flat-band geometry $[7]$ and exchange interaction anisotropy [8]. In the present study, we focus on the effect of interchain interactions, unavoidably present in a real compound. Although we bear in mind a spin model for azurite, we do not intend to explain this specific case but

*corresponding author; e-mail: derzhko@icmp.lviv.ua rather to consider theoretically a two-dimensional network of frustrated diamond spin chains with weak interchain interactions and to discuss the effect of the interchain coupling on localized-magnon features. To this end, we assume a simple geometry for interchain coupling proposed in Ref. [9] as shown in the upper part of Fig. 1.(Note, however, that this two-dimensional spin lattice cannot yield a consistent picture of experiments for azurite [5], rather it is adopted in our study only as a typical example.) In what follows we will elaborate an effective description of the model using the strong-coupling approach [10].

\section{Coupled frustrated diamond spin chains. Effective model}

To be specific, we consider the spin- $1 / 2$ antiferromagnetic $X X Z$ Heisenberg model of weakly coupled frustrated diamond chains in a magnetic field with the Hamiltonian

$$
H=\sum_{(i j)} J_{i j}\left(s_{i}^{x} s_{j}^{x}+s_{i}^{y} s_{j}^{y}+\Delta s_{i}^{z} s_{j}^{z}\right)-h \sum_{i=1}^{N} s_{i}^{z} .
$$

Here the first sum runs over all neighboring bonds of the $N$-site lattice, $J_{i j}>0$ is the antiferromagnetic interaction between the neighboring sites $i$ and $j$ and it acquires different values as shown in the upper part of Fig. 1 , and $0 \leq \Delta \leq 1$ is the $X Y$-like exchange interaction anisotropy. Moreover, we imply periodic boundary conditions.

Since the vertical dimer bond $J_{2}>0$ is the dominant one we may use the strong-coupling approach to obtain an approximate low-temperature high-field theory of the system under consideration. As a main system, we consider a collection of $\mathcal{N}=N / 3$ vertical dimers ( $J_{2}$ bonds) and $\mathcal{N}$ isolated sites labeled by $\boldsymbol{m}, 3$ at the "bare" saturation field $h_{0}=(1+\Delta) J_{2} / 2$ and denote its Hamiltonian as $H_{\text {main }}$. The rest of the Hamiltonian given in Eq. (1) (which contains the interaction terms $J_{1}, J_{3}$, and $J_{4}$ and 


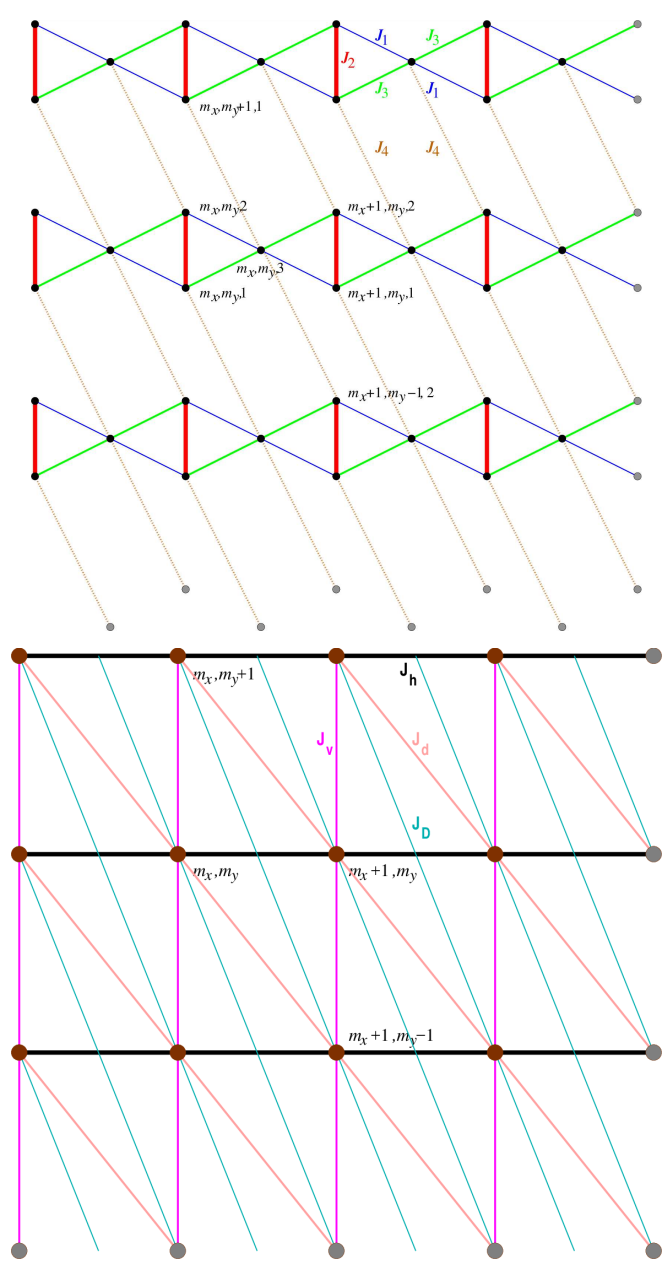

Fig. 1. Upper part: two-dimensional system of weakly coupled frustrated diamond spin chains, see Ref. [9]. $J_{2}$ is the strongest bond providing a trap for the localized magnon, $J_{1}=J_{3}<J_{2} / 2$ (ideal geometry) and $J_{1} \neq J_{3}, J_{1}+J_{3}<J_{2},\left|J_{1}-J_{3}\right| / J_{2} \ll 1$ (distorted geometry), and $J_{4}$ is the weakest bond connecting the chains. Lower part: sketch for a square-lattice (pseudo)spin-1/2 isotropic $X Y$ model in a transverse field given in Eq. (3) (effective model).

the Zeeman term with $\left.h-h_{0}\right)$ is treated as a perturbation $V=H-H_{\text {main }}$. In the high-field regime the dimers may be either in the state $|u\rangle=\left|\uparrow_{1} \uparrow_{2}\right\rangle$ or in the state $|d\rangle=\left(\left|\uparrow_{1} \downarrow_{2}\right\rangle-\left|\downarrow_{1} \uparrow_{2}\right\rangle\right) / \sqrt{2}$ (the energy of these states coincide when $h=h_{0}$ ) while the spins at the sites $\boldsymbol{m}, 3$ are in the spin-up state $|\uparrow\rangle$. Thus the ground state $\left|\varphi_{0}\right\rangle$ of $H_{\text {main }}$ is $2^{\mathcal{N}}$-fold degenerate at $h=h_{0}$. After switching on the perturbation $V$ we can construct perturbatively an effective Hamiltonian $H_{\text {eff }}$, which acts in the space spanned by $\left|\varphi_{0}\right\rangle$ only but gives the exact ground-state energy of $H$ [11]:

$$
H_{\mathrm{eff}}=P H P+P V \sum_{\alpha \neq 0} \frac{\left|\varphi_{\alpha}\right\rangle\left\langle\varphi_{\alpha}\right|}{\varepsilon_{0}-\varepsilon_{\alpha}} V P+\ldots,
$$

where $P=\left|\varphi_{0}\right\rangle\left\langle\varphi_{0}\right|$ is the projector onto the groundstate manifold of $H_{\text {main }}$ and $\left|\varphi_{\alpha}\right\rangle(\alpha \neq 0)$ are excited states of $H_{\text {main }}$. Finally, we may use the (pseudo)spin-
$1 / 2$ operators $T^{z}=(|u\rangle\langle u|-| d\rangle\langle d|) / 2, T^{+}=|u\rangle\langle d|$, and $T^{-}=|d\rangle\langle u|$ to rewrite the effective Hamiltonian in Eq. (2) in a better recognizable form.

After some straightforward calculations we arrive at the following effective Hamiltonian:

$$
\begin{aligned}
& H_{\mathrm{eff}}=\mathcal{N C}+\sum_{(m n)} \mathrm{J}_{m n}\left(T_{m}^{x} T_{n}^{x}+T_{m}^{y} T_{n}^{y}\right)-\mathrm{h} \sum_{m=1}^{\mathcal{N}} T_{m}^{z}, \\
& \mathrm{C}=-h-\frac{J_{2}}{4}+\Delta \frac{J_{1}+J_{3}+J_{4}}{4}-\frac{\left(J_{1}-J_{3}\right)^{2}+J_{4}^{2}}{4(1+\Delta) J_{2}}, \\
& \mathrm{~J}_{h}=\mathrm{J}_{m_{x}, m_{y} ; m_{x}+1, m_{y}}=\frac{\left(J_{1}-J_{3}\right)^{2}}{2(1+\Delta) J_{2}}, \\
& \mathrm{~J}_{v}=\mathrm{J}_{m_{x}, m_{y} ; m_{x}, m_{y}+1}=\frac{J_{4}\left(J_{1}-J_{3}\right)}{(1+\Delta) J_{2}}, \\
& \mathrm{~J}_{d}=\mathrm{J}_{m_{x}, m_{y} ; m_{x}+1, m_{y}-1}=-\frac{J_{4}\left(J_{1}-J_{3}\right)}{(1+\Delta) J_{2}}, \\
& \mathrm{~J}_{D}=\mathrm{J}_{m_{x}, m_{y}+1 ; m_{x}+1, m_{y}-1}=\frac{J_{4}^{2}}{2(1+\Delta) J_{2}}, \\
& \mathrm{~h}=h-h_{1}-\frac{\left(J_{1}-J_{3}\right)^{2}+J_{4}^{2}}{2(1+\Delta) J_{2}}, \\
& h_{1}=\frac{1+\Delta}{2} J_{2}+\Delta \frac{J_{1}+J_{3}+J_{4}}{2} .
\end{aligned}
$$

Here the first sum runs over the bonds on an auxiliary square lattice of $\mathcal{N}=N / 3$ sites (see the lower part of Fig. 1), $\mathrm{J}_{m n}$ acquires four different values, $\mathrm{J}_{h}, \mathrm{~J}_{v}, \mathrm{~J}_{d}$, and $\mathrm{J}_{D}$, see the lower part of Fig. 1. The obtained effective model is a square-lattice (pseudo)spin-1/2 isotropic $X Y$ model in a transverse field. Although the model contains four different exchange couplings, it is unfrustrated. In the limiting case when $J_{4}=0$ Eq. (3) coincides with the results obtained earlier $[7,8,10]$. In another limit when $J_{1}=J_{3}=J$ but $J_{4} \neq 0$ Eq. (3) yields $\mathrm{J}_{h}=\mathrm{J}_{v}=\mathrm{J}_{d}=0$ but $\mathrm{J}_{D} \neq 0$, i.e., one faces a spin- $1 / 2 X Y$ chain in a transverse field. This effective model is exactly solvable by Jordan-Wigner fermionization method. Finally, one can also consider the case when $J_{4} /\left|J_{1}-J_{3}\right| \ll 1$. Then $\mathrm{J}_{D}$ can be ignored and one faces a nonuniform triangularlattice spin-1/2 $X Y$ model in a transverse field.

To compare the initial model (1) with the effective model (3) we perform exact-diagonalization study for (periodic) finite systems. We consider the initial system of $N=36$ sites which correspond either to (i) 3 chains of 12 sites or to (ii) 4 chains of 9 sites. We consider several sets of parameters putting $J_{2}=3, J_{1}=J_{3}=1$ (ideal flat-band geometry), $J_{1}=0.85, J_{3}=1.15$ (distorted geometry), $J_{4}=0$ or $J_{4}=0.2$, and $\Delta=1$ or $\Delta=0$. We focus on the ground-state magnetization curves, see Figs. 2 and 3. As can be seen in Figs. 2 and 3 , the magnetization jump at the saturation - a prominent feature due to localized magnons [1] — is smeared out if $J_{4} \neq 0$. Effective-theory predictions for the region of steep increase of the magnetization around the saturation are somewhat underestimated. The agreement with the initial-model results becomes better when $\Delta \rightarrow 0$. Inspecting a pattern of spin correlations calculated for $N=3 \times 12(\Delta=1)$ at $h=0$ one concludes that the ideal geometry $J_{1}=J_{3}=1$ is more robust to the in- 


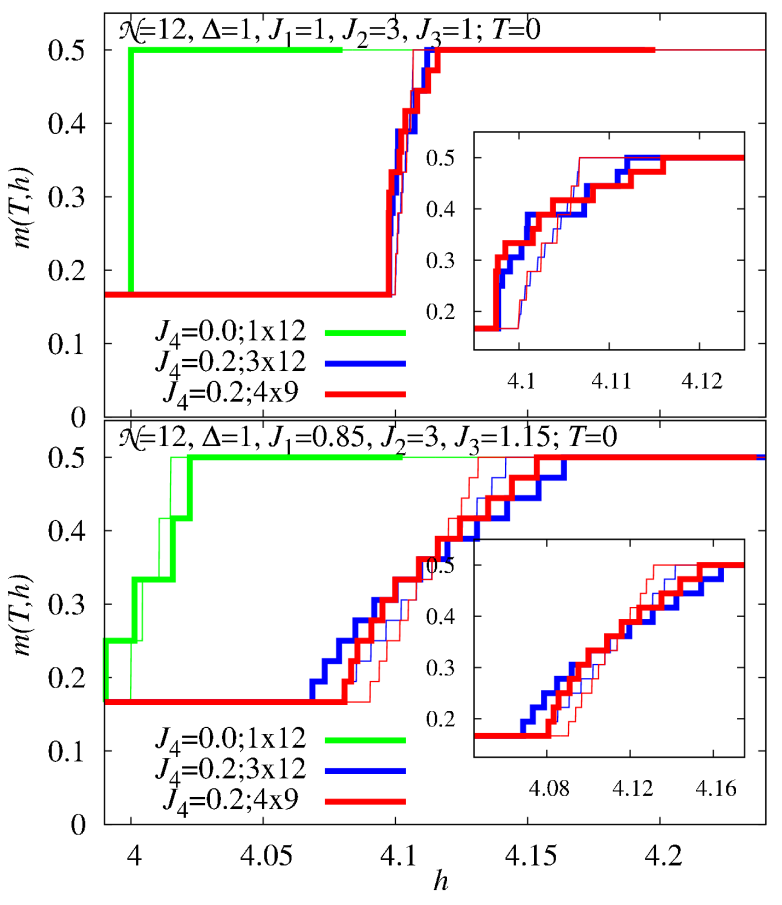

Fig. 2. Ground-state magnetization curves for the set of parameters $\Delta=1, J_{2}=3, J_{1}=J_{3}=1$ (ideal geometry, upper part), $J_{1}=0.85, J_{3}=1.15$ (distorted geometry, lower part), and $J_{4}=0$ (green) and $J_{4}=0.2$ (blue and red). Exact-diagonalization data refer to periodic systems of $N=36$ sites (initial model) or of $\mathcal{N}=12$ sites (effective model). Thick curves correspond to initial-model predictions. Thin curves correspond to effective-model predictions.

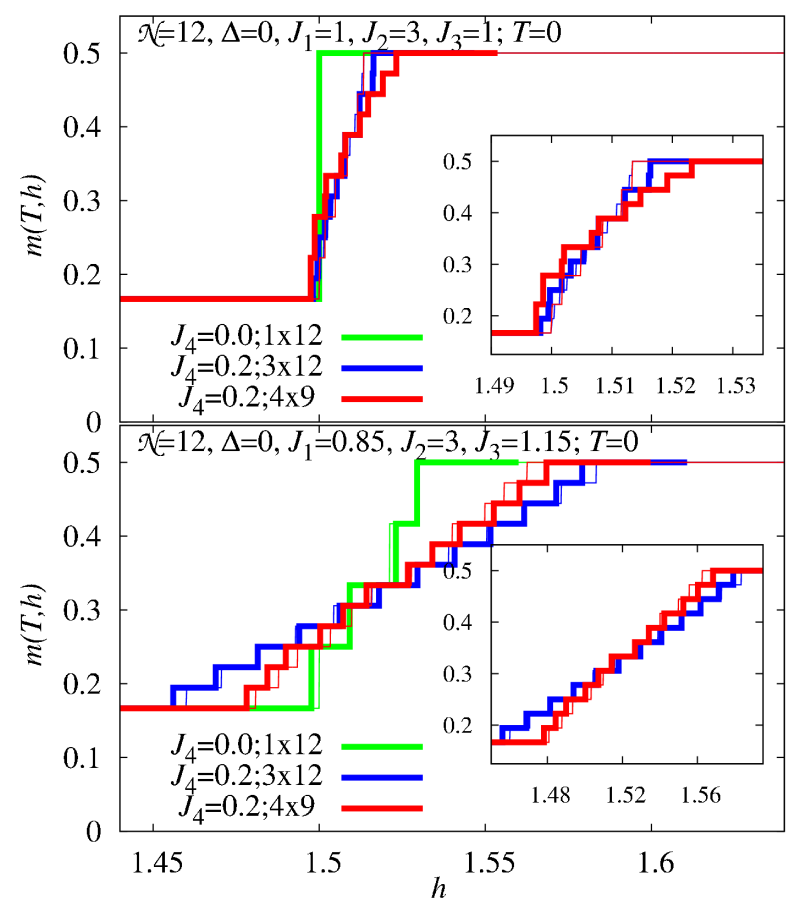

Fig. 3. The same as in Fig. 2, but $\Delta=0$. terchain coupling $J_{4}=0.2$ than the distorted geometry $J_{1}=0.85, J_{3}=1.15$.

\section{Conclusions}

In this paper, we examined a set of spin- $1 / 2$ frustrated diamond chains which support localized-magnon states in the presence of weak interchain couplings. Considering the case of low temperatures and high fields we used the strong-coupling approach and elaborated an effective (pseudo)spin-1/2 model on a square lattice. The effective model is unfrustrated and has three times less sites than the initial model. Thus, the effective model is simpler and can be studied by well known methods as, e.g., quantum Monte Carlo. Comparing the groundstate magnetization for the initial model and the effective one we observed a good agreement in the high-field regime. The effective model provides even better results if the anisotropy parameter for the initial model $\Delta$ approaches the $X Y$ limit. In general, the suggested approximation works reasonably well. However, from previous studies for the pure one-dimensional case $[7,8]$ we know that the accuracy of the strong-coupling approach becomes better if the vertical dimer bond $J_{2}>0$ increases. As to localized-magnon features, the interchain coupling washes out the remarkable ground-state magnetization jump at the saturation field. Finally, it is worthwhile noting that the discussed approach can be applied to other networks of coupled frustrated diamond spin chains.

\section{Acknowledgments}

The present study was supported by the DFG (project RI615/21-1). O.D. acknowledges the kind hospitality of the University of Magdeburg in March-May of 2016. The work of O.D. was partially supported by Project FF-30F (No. 0116U001539) from the Ministry of Education and Science of Ukraine. O.D. would also like to thank the Abdus Salam International Centre for Theoretical Physics (Trieste, Italy) for partial support of this study through the Senior Associate award.

\section{References}

[1] J. Schulenburg, A. Honecker, J. Schnack, J. Richter, H.-J. Schmidt, Phys. Rev. Lett. 88, 167207 (2002); J. Richter, J. Schulenburg, A. Honecker, J. Schnack, H.-J. Schmidt, J. Phys. Condens. Matter 16, S779 (2004); J. Richter, O. Derzhko, J. Schulenburg, Phys. Rev. Lett. 93, 107206 (2004).

[2] M.E. Zhitomirsky, H. Tsunetsugu, Phys. Rev. B 70, 100403(R) (2004); O. Derzhko, J. Richter, Eur. Phys. J. B 52, 23 (2006).

[3] O. Derzhko, J. Richter, M. Maksymenko, Int. J. Mod. Phys. B 29, 1530007 (2015). 
[4] H. Kikuchi, Y. Fujii, M. Chiba, S. Mitsudo, T. Idehara, T. Tonegawa, K. Okamoto, T. Sakai, T. Kuwai, H. Ohta, Phys. Rev. Lett. 94, 227201 (2005); H. Kikuchi, Y. Fujii, M. Chiba, S. Mitsudo, T. Idehara, T. Tonegawa, K. Okamoto, T. Sakai, T. Kuwai, K. Kindo, A. Matsuo, W. Higemoto, K. Nishiyama, M. Horvatić, C. Bertheir, Prog. Theor. Phys. Suppl. 159, 1 (2005); H. Kikuchi, Y. Fujii, M. Chiba, S. Mitsudo, T. Idehara, T. Tonegawa, K. Okamoto, T. Sakai, T. Kuwai, H. Ohta, Phys. Rev. Lett. 97, 089702 (2006).

[5] H. Jeschke, I. Opahle, H. Kandpal, R. Valenti, H. Das, T. Saha-Dasgupta, O. Janson, H. Rosner, A. Brühl, B. Wolf, M. Lang, J. Richter, S. Hu, X. Wang, R. Peters, T. Pruschke, A. Honecker, Phys. Rev. Lett. 106, 217201 (2011).

[6] A. Honecker, S. Hu, R. Peters, J. Richter, J. Phys. Condens. Matter 23, 164211 (2011).
[7] O. Derzhko, J. Richter, O. Krupnitska, T. Krokhmalskii, Phys. Rev. B 88, 094426 (2013); O. Derzhko, J. Richter, O. Krupnitska, T. Krokhmalskii, Fiz. Nizk. Temp. (Kharkiv) 40, 662 (2014) [ Low Temp. Phys. 40, 513 (2014)].

[8] J. Richter, O. Krupnitska, T. Krokhmalskii, O. Derzhko, J. Magn. Magn. Mater. 379, 39 (2015); O.M. Krupnitska, Ukr. J. Phys. 60, 1243 (2015).

[9] J. Kang, C. Lee, R.K. Kremer, M.-H. Whangbo, J. Phys. Condens. Matter 21, 392201 (2009).

[10] A. Honecker, A. Läuchli, Phys. Rev. B 63, 174407 (2001).

[11] P. Fulde, Electron Correlations in Molecules and Solids, Springer-Verlag, Berlin 1993, p. 77. 